Itinéraires Itinéraires

Littérature, textes, cultures

2019-2 et $3 \mid 2019$

Corps masculins et nation : textes, images, représentations

\title{
A man's world : incorporation langagière à la Légion étrangère
}

A Man's World: Language Incorporation in the French Foreign Legion

\section{Mélanie Texier}

\section{OpenEdition}

\section{Journals}

Édition électronique

URL : http://journals.openedition.org/itineraires/6306

DOI : $10.4000 /$ itineraires.6306

ISSN : 2427-920X

Éditeur

Pléiade

Référence électronique

Mélanie Texier, " A man's world : incorporation langagière à la Légion étrangère », Itinéraires [En ligne], 2019-2 et 3 | 2019, mis en ligne le 29 novembre 2019, consulté le 15 décembre 2019. URL : http:// journals.openedition.org/itineraires/6306; DOI : 10.4000/itineraires.6306

Ce document a été généré automatiquement le 15 décembre 2019.

\section{cc) (1) $९$}

Itinéraires est mis à disposition selon les termes de la licence Creative Commons Attribution - Pas d'Utilisation Commerciale - Pas de Modification 4.0 International. 


\title{
A man's world : incorporation langagière à la Légion étrangère
}

\author{
A Man's World: Language Incorporation in the French Foreign Legion
}

\author{
Mélanie Texier
}

1 Aucun texte officiel n'interdit de recruter des femmes à la Légion étrangère depuis sa création $^{1}$. La condition initiale est en effet d'être étranger. Pourtant, elles en sont absentes et la Légion étrangère reste un pré carré masculin dont la représentativité virile n'a été affectée ni par la professionnalisation des armées et l'ouverture du métier militaire aux femmes, ni par la suppression de quotas à un grand nombre de postes à l'aube des années $2000^{2}$. Par représentation virile, j'entends une «image mentale stéréotypée » qui lie le corps et l'esprit comme un tout indissociable et perçue comme une unité équilibrée (Moss 1997 : 11) dans une optique phénoménologique. C'est de même en ce sens que j'emploie les termes de corps masculins et de corps féminins. La matérialité des corps introduit dans les représentations du corps militaire comme corps professionnel culturé des rapports de sexage qui passe par une fonction des corps comme premier indice de sexe (Guillaumin 1992: 117), actualisés socialement, langagièrement. Cette approche permet par ailleurs de lier les discours de nature sur les pulsions masculines et les discours de culture sur la force masculine dans lesquels, alors que l'image de la virilité est bien celle de la maîtrise de soi dans les armées, on considère que les hommes ne peuvent pas contrôler leurs pulsions sexuelles (Héritier 2011 : 23). Prendre comme point d'appui le corps est donc à la fois partir de sa matérialité pour atteindre son immatérialité par un feuilleté langagier de ce même corps et déconstruire ainsi leur richesse sémantique particulièrement en œuvre dans les armées : esprit de corps, corps d'armée, corps de troupe, corps des officiers, corps de bâtiment, chef de corps, repas de corps ${ }^{3}$, etc. C'est aussi mettre en tension épistémologiquement ces mêmes représentations en jeu dans la construction d'une identité professionnelle militaire.

2 La Légion étrangère reste de fait un monde d'hommes, une culture militaire agnatique fondée sur une cohésion horizontale de fraternité et une autorité verticale paternaliste. La patrie n'y est pas la France mais bien la Légion étrangère comme le signale sa devise 
«Legio patria nostra». Quant à la femme, c'est l'incorporation militaire ${ }^{4}$ qui la consacre comme objet langagier : fantasmée, sexualisée, frontiérisée, étrangère.

3 La fabrication d'une identité militaire dépasse en effet la construction professionnelle. L'incorporation militaire était lors du service militaire vécue comme un rite de passage pour les hommes et marquait le début de l'âge adulte. Elle ne transformait pas ainsi seulement des individus en soldats, elle en faisait des hommes. La professionnalisation des armées à l'aube des années 2000 et l'ouverture du recrutement aux femmes n'a eu que peu d'impact sur ce rôle symbolique des armées. Ce rite de passage de la masculinité perdure et inscrit un sexe de la guerre dans chaque homme en uniforme. Cette inscription se retrouve de surcroît dans chaque corps féminin engagé dans un corps d'armée. Conjointement, la socialisation professionnelle fonde un corps militaire transcendant les individualités, un corps collectif où chaque sous-culture de l'armée de Terre a ses spécificités, à la recherche d'un esprit de corps surpassant tous les autres.

4 Considérer que, parce qu'il n'y a pas de femmes à la Légion étrangère, il n'y a pas de problèmes d'égalité professionnelle, de féminisation d'un corps militaire - ou au contraire qu'il s'agit là d'un terrain symbolique des plus grandes inégalités - n'est en réalité qu'un fait de surface. La défense d'une spécificité de la Légion étrangère est en effet acceptée par l'ensemble des armées et érige la Légion comme un bastion masculin à préserver, le dernier à pouvoir tomber d'une conquête féminine des armées.

5 Afin de comprendre la construction d'un corps militaire collectif réifié dans chaque corps masculin, j'ai articulé le questionnement de ces corps à deux corpus imbriqués qui façonnent un corps discursif. En premier lieu, les carnets de chants édités depuis 1959 par la Légion étrangère sont interrogés comme des prédiscours (Paveau 2006) qui orientent les pratiques langagières des légionnaires et fournissent des savoirs langagiers, notamment lors de l'incorporation sur l'identité collective. Je propose ici une sélection de ces analyses qui questionnent non seulement les représentations sur les femmes, mais aussi et surtout leurs fonctions dans la guerre. En second lieu, des entretiens menés entre 2012 et 2016 auprès d'officiers de légion explorent une incorporation langagière où les corps s'enchevêtrent entre matérialité et immatérialité langagière pour constituer un discours sur le corps militaire. Entre normes institutionnelles et appropriations groupales, ces discours mettent ainsi au jour la naturalisation du corps combattant, alors même que les armées disciplinent les corps en soumettant les passions et les pulsions à l'autorité et à l'ordre, de sorte que "l'image de la virilité est bien celle de cette maîtrise de soi » (Héritier 2011 : 23).

\section{L'incorporation des pratiques langagières}

6 Dans mes recherches sur la Légion étrangère ${ }^{5}$, j'en suis venue à me saisir de l'incorporation, qui acte l'engagement militaire et le début de la formation, pour désigner la socialisation professionnelle des militaires. J'appelle incorporation un double processus langagier qui intervient dans la médiation d'une identité en construction. D'une part, il implique une phase d'intériorisation, où les normes et les règles d'un groupe imprègnent les corps. D'autre part, il nécessite une phase de recomposition de cette intériorisation, pour inscrire l'identité d'une personne en cohérence avec son parcours de vie. Appréhender cette incorporation comme un processus langagier consiste de plus à lier l'immatérialité du langage à la matérialité d'un corps. Dans cette optique, le langage joue ce rôle essentiel de nous mettre au monde dans nos rapports 
intersubjectifs tout comme le corps est un naître/être au monde (Merleau-Ponty [1945] 1997 : 515).

7 L'incorporation repose sur cinq propriétés qui sont les conditions de sa réalisation: l'adhésion (1), en tant que libre soumission à un ordre social ; l'expérientialité (2) qui s'ancre dans chaque corps comme vécu, expérience de vie et prend appui sur les rites et la mémoire qui inscrivent un faire faire et un faire dire les transcendant; l'intersubjectivité (3) d'un individu jeté dans le monde et confronté à l'autre où on incorpore son semblable générant une frontière phénoménologique et discursive entre un nous et des autres qui deviennent étrangers, même s'ils restent potentiellement incorporables; la matérialité (4) permet de projeter le corps dans des objets qui revêtent une matérialité symbolique comme objets langagiers et qui narrent autrement la relation au monde d'un groupe (monuments aux morts, drapeaux, tatouages, etc.) ; la discursivité (5) réside dans l'interdépendance entre discours et pratiques langagières et interroge les prédiscours comme "cadre de savoir et de croyance qui informent directement les discours produits» (Paveau 2006: 118). La discursivité est donc le dernier acte de l'incorporation, comme appropriation où les discours produisent des pratiques langagières et où les pratiques langagières produisent des discours. Le code d'honneur des légionnaires, comme tout code militaire et toute charte déontologique organisationnelle, produit ainsi un discours normatif sur l'organisation, dont chaque individu ne se saisit pas uniformément, mais où il s'approprie ce qui est nécessaire à son identité groupale et ce qui est cohérent avec son parcours de vie, dépassant le cadre de l'engagement professionnel pour s'intriquer avec l'ensemble des facettes de son identité. La problématique de la Légion étrangère est d'ajouter à la discipline des corps en formation l'apprentissage du français, comme langue de travail en partage. Plus de 140 nationalités ${ }^{6} s^{\prime} y$ rencontrent ainsi et sa richesse plurilingue est assujettie à une norme langagière monolingue par le creuset du régiment-école, le $4^{\mathrm{e}}$ régiment étranger. L'ensemble participe de la création de la cohésion groupale et d'un esprit de corps qui :

[...] unit les soldats autour d'une cause qui les dépasse. Il est fait d'adhésion, d'attachement à une identité collective puissante, de solidarité, de dévouement, de dépassement de chacun dans un " être collectif ». Le soldat le crée et le fait vivre, en hérite et le transmet. Historiquement forgé dans les situations de combat, il prépare, il irrigue l'environnement opérationnel. (État-major de l'armée de Terre $2003: 7)$

8 Lors de son engagement, le légionnaire reçoit en effet le Code d'honneur du légionnaire, instauré dans les années 1980 comme «référence morale et règle de comportement à tenir au quotidien et lors des combats » (COMLE 2010), ainsi qu'un carnet des chants de la Légion étrangère. Le chant militaire intervient comme soutien à l'effort pendant une marche ; comme événement de cohésion lors de la popote, terme d'argot militaire qui désigne un repas de cohésion; comme moyen d'expression et de revendication qui contourne le devoir de réserve et évolue en marge des règlements militaires. Le TTA ${ }^{7}$ 107 qui existe depuis 1985, suite à une première version établie en 1980 où l'institution militaire s'est saisie des chants pour les normer et les faire "rentrer dans le rang", indique ainsi que le chant est " la première manifestation de la cohésion d'un groupe " (État-major de l'armée de Terre 1985, n. p.). Ils sont ainsi un lieu de dépôt identitaire où sont affirmées les valeurs militaires transmises de génération en génération de soldats et où les appropriations successives participent aussi de l'évolution du répertoire (Thiéblemont 1999: 259). De fait, ils sont une réserve identitaire active (Paveau 2000 : 
259) qui rend compte à la fois de l'incorporation, comme intériorisation des normes et appropriation de celles-ci afin de restituer une pratique langagière collective qui est un discours sur ce que sont les corps militaires, et des valeurs du corps combattant. Enfin, cette incorporation est projetée matériellement car le chant est articulé aux lieux et aux espaces dans lesquels les légionnaires évoluent à différentes échelles : un espaceLégion d'appartenance pour Le Boudin, géographique et de creuset commun de formation pour celui du $4^{\mathrm{e}} \mathrm{RE}$, humain pour ceux de la compagnie et de la section; autrement dit, un espace-Légion d'appartenance collective, un espace discursivement générateur de l'identité collective et un espace matérialisé de l'identité collective accessible par une échelle groupale à taille humaine et brassant une réalité expérientielle.

\section{L'adhésion comme renoncement de soi}

9 J'ai ainsi constitué un corpus de 57 chants issus de l'ensemble des carnets de chants édités depuis 1959, à partir des versions ici présentées de 1959, 1973, 1982, 1985, 1989, 1993, 1998 et 2011. Je n'ai pas retenu les carnets de chants édités par des unités, des régiments particuliers ou relevant de distinctions hiérarchiques pour me concentrer sur ceux édités officiellement comme discours que tient la Légion étrangère sur son identité militaire. Les chants composant ce corpus sont tous en langue française, le plurilinguisme des chants ayant fait l'objet d'une analyse spécifique. Afin d'éviter de produire des préconçus sur ces chants - le combat, l'ennemi, les femmes, l'alcool, etc. et de ne pas considérer que ces chants avaient déjà tout dit (Pétonnet 1982:39), j'ai formulé dans un premier temps deux hypothèses: premièrement, le chant a des fonctions cohésive et généalogique comme support différencié d'une armée plurielle ; deuxièmement, ces fonctions, comprises dans un ensemble nommé traditions dans les armées, ne sont qu'un premier feuilleté qui dissimule une dimension organisationnelle normative. La première hypothèse, qui n'est pas novatrice (Paveau 1999, Poussin 2014), a été l'occasion d'y déceler les représentations identitaires partagées par l'ensemble de l'armée de Terre voire des armées à travers l'appartenance institutionnelle de la Légion étrangère. Je me suis donc davantage concentrée sur la seconde hypothèse que je restitue ici.

Dans un second temps, j'ai ainsi pris le parti d'appliquer une observation flottante (Pétonnet 1982) à ces chants pour ne pas les interpréter selon mes propres préconçus. Puis, j'ai utilisé le logiciel IRaMuteQ, envisagé comme un biais heuristique afin de dégager des mondes lexicaux comme "trace du monde propre d'un sujet énonciateur, trace des objets qui sont à la fois "objets du monde" et "cibles" de son activité " (Reinert 1983: 14). Bien entendu, ces traces sont ici celles d'un sujet-énonciateur collectif qui met en valeur un nous nostro-centrique (Kaufmann 2006 : 101), celui de la collectivité et de fait anonyme.

11198 segments à partir des formes actives ont ainsi été classés sur les 220 détectés par IRaMuTeQ $Q^{8}$, soit $90 \%$, et cinq classes ont été mises à jour en supprimant les classes 0 , c'est-à-dire celles qui n'ont pas été retenues par le logiciel. Je m'arrêterai dans cette analyse des représentations du corps masculin sur les classes 1 et 5 . 
Fig. 1. Classes établies par le logiciel IRaMuTeQ à partir des carnets de chants de la Légion étrangère

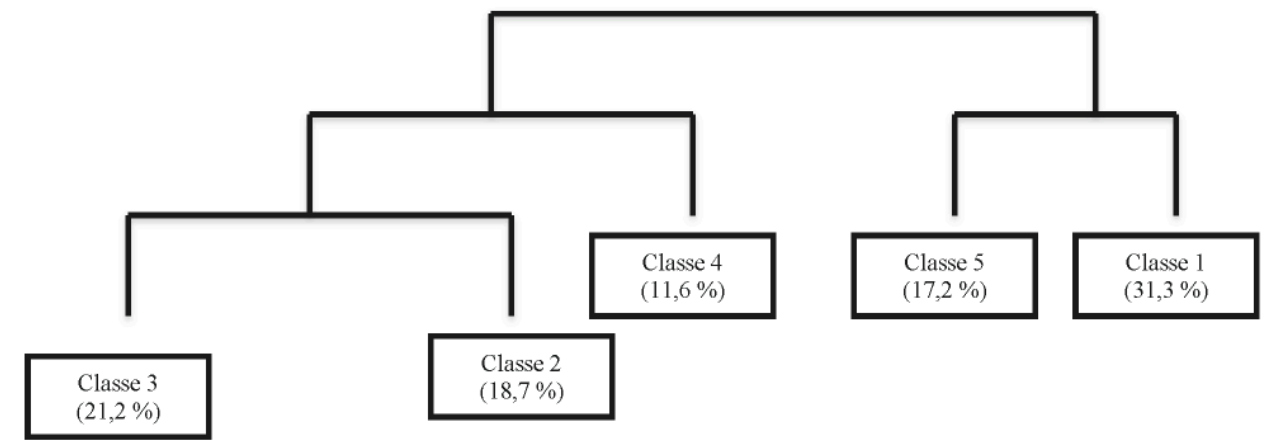

La classe $1^{9}$ est celle de l'abandon de la femme et de la valorisation du célibat, condition d'engagement contractuel à la Légion étrangère. L'identité personnelle est ici matérialisée par des marqueurs environnementaux comme la berge, la barque, qui peuvent à leur tour convoquer des souvenirs. Ces lemmes sont renforcés par des adjectifs qualitatifs comme joli, beau ; certains expriment l'immaturité comme jeune ou petit, soulignant tout autant la jeunesse des individus que leur inexpérience :

On lui dit viens petit chez nous chercher l'oubli

(Le Fanion de la Légion, Carnet de Chants et Marches de la Légion étrangère)

Lorsque j'étais petit, je croyais qu'un démon

Viendrait me ravir à ma maison,

Mais lorsque je fus grand,

Ce fut une horrible guerre,

Qui m'emmena loin de mes terres

(Massari Marie, Carnet de Chants et Marches de la Légion étrangère)

L'identité personnelle appelle ainsi à une rupture avec son passé et à un abandon de la femme pour que l'homme mûrisse à partir de son incorporation militaire. Les femmes justifient l'engagement et l'engagement justifie l'abandon des femmes, qui n'ont pas de place à la guerre :

Quand on a une fille dans le cuir et que la vie vous dégoûte...

(Quand on a une fille dans l'cuir, Carnet de Chants et Marches de la Légion étrangère)

Au revoir petite Monica,

Nous partons au combat,

Au revoir, petite Monica,

Que tes yeux ne pleurent pas

(Monica, Carnet de Chants et Marches de la Légion étrangère)

Sur toutes les routes y a du chagrin

(Les Cailloux, Carnet de Chants et Marches de la Légion étrangère)

Les verbes qui appartiennent à cette classe vont d'ailleurs dans ce sens avec des marqueurs émotionnels de pathos comme pleurer, des marqueurs de l'arrachement comme partir, perdre. Ils soulignent la contingence de cette vie par le biais de verbes comme oublier ou se foutre: «je me fous du passé»; ou renvoient à la dimension éphémère de l'existence avec passer, par exemple.

La femme est toujours seule dans ces chants, comme Frederi en larmes sur une berge et dont l'abandon est souligné par un anneau d'or perdu, symbole du mariage qui n'aura pas lieu, ou encore Marie pleurant son homme parti : 
Mais les mois et les années passèrent,

La Marie a pleuré bien souvent

En songeant aux beaux jours de naguère [...]

[...] La Marie qui était si jolie

A perdu sa beauté de vingt ans,

Quand on pleure, on vieillit, c'est la vie,

Ses grands yeux sont tout gris à présent

(Le Gars Pierre, Carnet de Chants et Marches de la Légion étrangère)

On retrouve par ailleurs à de nombreuses reprises le verbe penser : je pense à toi, penser à l'amour, penser à la femme; mais cette pensée est éphémère et ne dure pas, contrebalancée encore une fois par des verbes de l'oubli : oublier, passer, perdre. L'oubli lie donc la femme et l'engagement. Il est un des marqueurs discursifs de la Légion, prépondérant dans les discours sur l'engagement des légionnaires et l'hospitalité de l'accueil comme droit à l'oubli : «Certains légionnaires parlent de fuite. Ils expliquent leur attitude par un besoin impérieux de se soustraire aux responsabilités dont toute une vie est composée : métier, vie conjugale, paternité » (Montety 2006 : 19).

Dans les discours des officiers, l'adhésion est divisée en strates concentriques : liberté d'engagement, volontariat, motivation puis renoncement de soi et don total de soi incluant le sacrifice de sa vie. Le renoncement de soi serait alors un dépôt provisoire de son corps pour habiter un autre corps : celui du collectif, celui du corps de métier. Yves Lichtenberger (2014: 99), sociologue du travail, met en parallèle le renoncement et le compromis à la suite de Christian Thuderoz (2013: 97), qui introduit l'idée que le compromis est un renoncement partiel et mutuel pour atteindre un résultat supérieur, où abandonner est concéder. Par le compromis, les individus se défont de leur capacité d'agir, moteur de la liberté et du volontariat, car si le compromis coordonne, il n'inspire pas d'actions, il est un référentiel bricolé pour que « les cours d'action sociale parviennent, enfin, à leurs objectifs - et que vivre ensemble soit possible » (Thuderoz 2013 : 101). Les individus renoncent en ce sens par compromis aux femmes :

HCSCO1-18 $8^{10}:[.$.$] c'est c'est un vrai parcours et c'est pas facile donc c'est est-ce que$ c'est une clôture ou un espace préservé le but c'est pas de les enfermer c'est de les aider à en sortir mais pour les aider à en sortir il faut un peu de méthode il faut un peu de repères avant qu'ils soient autonomes puisque progressivement dans leurs parcours ils vont ben avoir plus d'autonomie avoir une situation juridique plus simple ils vont non plus vivre au quartier ni dans une caserne mais aller vivre à l'extérieur ils vont se marier avoir des enfants construire voilà :/ bon bah tout ça ça passe par:/ cette période exigeante de renoncement de soi pour le bien de la Légion. HCSC01-50: [...] bon il s'est engagé à dix-sept ans dix-huit ans il a fait cinq ans en Algérie euh::: il avait une: voilà il a voulu prouver à sa copine que: euh il pouvait voyez donc souvent c'est ça c'est un renoncement pour mieux revenir c'est une coupure voyez enfin toutes les postures masculines qu'on peut imaginer dans la relation avec les femmes on les trouve exacerbées chez le légionnaire.

(Entretien mené avec un officier de Légion en 2016)

\section{Exacerbation du collectif et sexualisation du corps combattant}

La femme abandonnée est réinvestie comme un "bien commun » approprié par le groupe où se profile un «caractère naturel » (Guillaumin 1992: 14) et attachée à l'expérientialité d'un corps formé et incorporé. La « fille dans le cuir » qui pousse à 
l'engagement devient une fille, substitut d'amour dont le nom propre disparaît pour n'être plus qu'une figure sexualisée anonyme pour un corps collectif :

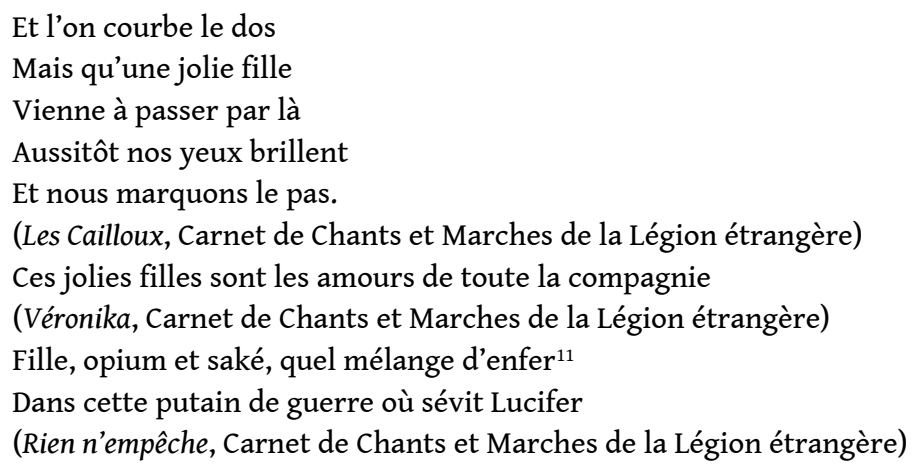

Si Aage du Danemark a écrit que "sans les femmes il n'y aurait pas de Légion étrangère " (Montagnon 2006: 36) et que Marie Larroumet confirme leur présence, réinvestie dans le quotidien militaire (féminisation des noms de jeeps, des points d'appui, des animaux de type mascottes, etc.), elle précise par ailleurs que la Légion étrangère entretient des rapports certes fréquents mais " complexes" avec les femmes (Larroumet 2004 : 97). Cette ambivalence apparaît ici entre une femme aimée et une femme à aimer, où séduction et sexe s'enchevêtrent entre éphémérité et permanence. Ce rapport aux femmes, d'une part, la minore socialement en marquant l'attraction de l'engagement, où la femme est celle qu'on laisse pour partir et celle qu'on retrouve au retour. D'autre part, elle est minorée sexuellement, assignée à être un objet de désir collectif. La guerre recompose la place sociale des femmes, qui passe ainsi d'une dimension individuelle à collective sur le temps de l'engagement, puisque la forme revenir suggère un basculement à nouveau de la norme collective vers la norme individuelle. Colette Guillaumin aborde ce passage de l'individu au collectif mutatis mutandis dans les rapports de sexage par les différentes expressions d'appropriation: l'appropriation du temps, l'appropriation des produits du corps ; l'obligation sexuelle ; la charge des membres invalides du groupe et des membres valides de sexe mâle (Guillaumin 1992 : 20). La guerre modifie en effet les rapports d'appropriation dans le sens où le combattant peut potentiellement s'approprier tous les biens matériels de l'ennemi. Les femmes sont donc comprises dans cette optique comme un bien matériel que les soldats s'approprient et se partagent. En temps de paix, les appropriations ne s'expriment pas également mais perdurent et restent mobilisables. Gaston Bouthoul, à la suite de Clausewitz, considère ainsi que la guerre est un duel qui structure les représentations entre ami et ennemi (Bouthoul 1970 : 328). Ces représentations sont le réceptacle énonciatif de l'ensemble des représentations qui donnent corps à la doctrine militaire (Péries 1999 : 289) à envisager comme le discours que produisent les armées sur leur rôle, leur fonction, etc. Tarde ([1890] 1993) inscrit de plus le duel qu'il nomme logique comme le premier germe de la guerre, de l'opposition sociale. J'ai proposé dans mes travaux de thèse qu'en temps de paix ami et ennemi soient substitués par ami et concurrent. Les femmes restent donc exclues de ces représentations mais en sont néanmoins de même toujours l'objet. L'appropriation en temps de paix revêt donc les habits de la séduction et est un indice d'une conquête virile marquant une militarité normée par la dimension combattante. Les rapports inégalitaires entre les hommes et les femmes sont par conséquent des rapports de pouvoir tels qu'on les retrouve dans les sociétés. $Y$ ajouter une dimension militaire, c'est inscrire sur un phénomène social violent des rapports de pouvoir qui assujettissent d'autant plus les femmes en les 
assignant à être des objets. Leur absence ou plutôt leur extériorisation des frontières discursives d'une militarité en construction s'inscrit donc dans cette appropriation des corps féminins à mesure que les corps masculins se rassemblent en un corps professionnel lié par la cohésion. Le célibat n'est donc plus une condition, il est désormais un état du légionnaire. On peut être "célibataire géographique" dans les autres armées, catégorie lexicale marquant l'éloignement de la famille comme lieu de résidence. À la Légion étrangère, il n'existe pas de catégorie lexicale intermédiaire : la famille est la Légion étrangère et c'est ce qui apparaît ici dans les chants. Cela ne signifie nullement que le légionnaire n'a pas de famille, ou que ces familles institutionnelles et sociales s'ignorent, mais la Légion étrangère supplante les normes sociales traditionnelles pour les refondre en normes fonctionnelles, car elle a besoin de combattants pouvant sacrifier leur vie. Et nécessairement, il est plus facile de donner sa vie quand on n'a rien à perdre et personne pour pleurer ces morts et les réclamer. Cette dimension est plus souvent entendue qu'explicitée par l'organisation. Elle est facilitée à la Légion étrangère par le statut d'étranger qui éloigne les familles et limite les possibles contacts pouvant s'établir avec elles.

La classe 5 qui est directement liée à la classe 1 pour former ainsi une branche du dendogramme est celle du temps organisationnel militaire, de l'attente et de la préparation. Elle marque le glissement de l'engagement à l'attente pré-opérationnelle des militaires. Il ne s'agit plus dans la classe 5 de conditions de réalisation en vue d'une incorporation mais d'une expérentialité qui n'est pas marquée par les combats, l'action. L'analyse de la classe 5 met en évidence la saillance du lien plus fort entre képi blanc, qui désigne le légionnaire, et aller d'un côté, attendre de l'autre. L'attente est ainsi fléchée dans deux directions: l'une en direction du combat et l'autre liée à la figure de la femme. Celle-ci est ainsi désormais liée exclusivement à l'attente, où le temps qui lui est consacré s'inscrit dans celui qu'autorise le temps militaire ou qui est pris sur le temps d'attente militaire. La forme serment caractérise autant les serments faits à la femme que le serment contracté à l'engagement. Toutefois, le pluriel s'inscrit en contraste de l'unique serment fait à la Légion étrangère et marque une nouvelle fois la prégnance de l'engagement militaire face à tout autre engagement. Le second temps d'attente est lié au combat. La forme ennemi est ainsi considérée comme non significative par IRaMuteQ mais contextuellement, elle explicite l'attente du combat et je l'ai donc inclus dans mon analyse:

Je vais où le baroud m'attend.

C'est mon devoir de faire la guerre,

Partout où l'ennemi m'attend

(Cravate verte et képis blancs, Carnet de Chants et Marches de la Légion étrangère)

L'attente est donc une attente opérationnelle, marquée par l'incertitude et l'attente du combat à venir projeté sur une spatialité inconnue, tandis que les femmes sont un réinvestissement par les légionnaires du temps d'attente. L'attente, comme le souligne Thura (2014: 58), fait « partie du boulot... elle est un effet induit par l'organisation du travail militaire ", soit parce que l'unité n'est qu'un élément d'un dispositif plus large qui est en attente de coordination et d'ordres par transmission descendante, soit parce que, et c'est le cas ici, le temps est soumis à un tiers (l'ennemi) où il s'exprime tactiquement par son imprévisibilité et l'effet de surprise. Le temps est donc à la fois caractérisé par son incommensurabilité comme fuite ou étirement et l'action, ellemême, est mise en tension par un agir sur l'attente. Cet agir implique une maîtrise de 
soi, bien qu'on ait la rage aux dents: il faut donc serrer les dents en contrôlant ses passions et ses pulsions. La femme devient alors, dans l'attente, l'expression de passions et pulsions qui peuvent être enfin libérées par son objectivation en figure sexualisée. Elle est attente, elle est un dérivatif à l'inaction et une action contre l'attente.

La représentation langagière de la femme l'érige alors comme femme-objet passive dans un contexte discursif où le corps collectif détient tout agir langagier et compétence par la guerre à s'imposer physiquement: baiser, se faire sucer le noeud, se faire sucer le poireau sont ainsi mis en parallèle avec d'autres usages argotiques comme moukères, gonzesses. Les femmes ne sont plus à aimer, elles sont objet de compétition afin d'entretenir la pugnacité des hommes :

Les femmes, et les fiancées! Qui c'est qui s'les tape

(Susanna, Carnet de Chants et Marches de la Légion étrangère)

Ce paradigme de la femme-objet est de fait lié au boire soldatesque et à un lexique argotique de l'alcool : pinard, chiquer une anisette, bistrot. Le boire soldatesque est ainsi associé au combat comme dérivatif, de même que la femme est un dérivatif dans l'attente du combat. Elle est par conséquent une combativité de loisirs : «on boit dans sa perspective angoissante, pour surmonter son imminence et s'exalter avant d'affronter l'ennemi, on s'enivre pour fêter la victoire ou encore pour oublier l'horreur des combats, enfin on se grise pour s'occuper en son absence » (Prévot 2007 : 160). Dans Adieu Diégo, la première strophe renvoie ainsi à l'alcool et les deuxième et troisième aux prostituées. Dans Pour faire un vrai légionnaire, on peut lire :

[Première strophe]

Chacun sait bien qu'un Légionnaire

est le premier dans les combats,

Que sa bravoure est légendaire [...]

[Dernière strophe] : [...] On ne peut pas leur faire le coup,

Ce sont des femmes de Légionnaires

Elles veulent qu'on soit premier partout.

\section{Naturalisation ${ }^{12}$ des corps par la guerre}

L'incorporation des pratiques langagières d'une identité légionnaire réifie une masculinité guerrière qui évacue toute représentation féminine d'un corps exclusivement masculin et la relègue à la périphérie des combats comme un être en attente, "à l'arrière ", ou un objet sexuel collectif, dérivatif d'une attente des combats et de la puissance guerrière. La violence guerrière ramène toujours à une histoire du corps (Audoin-Rouzeau et Becker 2000:24-26) et à un corps masculin, qu'il soit individuel ou collectif. Dans les entretiens avec les officiers de Légion, discours de la nature humaine et discours de la culture militaire sont intriqués, donnant lieu à une nature de la guerre. Mêler la nature et la culture dans un contexte militaire impose de prendre en considération que la nature implique une nature humaine considérée comme un état individuel. Cette nature humaine précède une entrée en société alors que la nature de la guerre superpose en fait une culture marquée par une capacité naturalisée de violence dans la guerre. En prenant la vie, en la défendant par son intervention, le militaire est un régulateur de l'écosystème humain; il remplit « une fonction nécessaire au bon accomplissement du processus vital ", tout en exprimant à travers la guerre " une sensibilité vraie " (Gresle 2003: 783) où il révèle non pas une condition militaire mais un état militaire, mobilisable en tout individu. L'institution 
militaire augmente l'individu considéré comme fragile pour le constituer en corps collectif fort et solide, apte à démontrer sa virtus, sa virilité :

SCC05-23 : c'est un étranger:/ dans la plupart des cas :/ euh : au service de la Légion étrangère d'abord de la France par :/ par ricochets par corrélation :// assez instable c'est une forme de fragilité:/ individuelle mais qui mis euh: au service d'un collectif très fort en fait une formidable machine de guerre au sens positif du terme. SCC05-29: [...] la Légion étrangère elle est forte que collectivement individuellement le légionnaire il a des fragilités comme je le disais tout à l'heure donc en fait non ça fonctionne bien ça fonctionne plutôt bien et c'est une nécessité. SCC02-76: euh : ça et puis après la cohésion je pense surtout à la Légion c'est :/ le gros défi c'est de niveler les différences :/ pour justement arriver à un groupe euh le plus homogène possible:/ niveler les différences justement ça passe par les traditions qui sont mises en place :// le système qui est mis en place par la Légion on parlait de la langue on parlait de l'identité on parlait aussi d'excellence opérationnelle de fierté donc voilà la cohésion après c'est transformer des individus en un bloc qui dépasse euh ses euh : ses propres différences quoi pour justement finalement arriver à la force du groupe donc c'est-à-dire rabaisser en quelque sorte les meilleurs les mettre au service des plus faibles pour que justement le groupe s'élève.

SCC03-94: [...] c'est la force de la Légion c'est de justement réussir à faire un amalgame et à la rendre homogène euh :: grâce à cette cohésion on arrive à avoir ----interférences---- euh ::: mais on a pas forcément que des types virils:/ mais vu qu'ils se retrouvent dans un milieu euh :: je dirai qu'ils se qu'ils cherchent peut-être à dépasser leurs limites

(Entretiens menés auprès d'officiers de Légion en 2012)

La convocation de la nature renvoie à la fois à l'adhésion des légionnaires comme ce qui va de soi par le volontariat, et à l'acceptation des règles hiérarchiques qui prévalent à la Légion étrangère comme données d'emblée à tous, militaires du rang, sous-officiers et officiers. La socialisation secondaire reprend de fait les caractéristiques d'une définition de ce qu'est la nature: ce qui est donné dès le départ, qui permet de répondre à des besoins naturels pour refonder un état de nature modelé par l'institution militaire. Dans un sens hobbesien, quitter son état de nature serait donc quitter son individualité pour faire société, s'engager dans une société. L'engagement militaire est donc une réponse (tout autant qu'une proposition) à la fragilité individuelle où en faisant société, l'individu incorpore la force groupale. Le groupe est ainsi associé à l'idée de force (Maisonneuve [1968] 2018) où chaque individu est augmenté de la masse des autres, jusqu'à faire corps, et reflète la solidité du groupe. Les lexèmes employés pour qualifier les groupes militaires vont d'ailleurs en ce sens et soulignent la pluralité dans une unité solide: la phalange se réfère au corps en désignant les petits os qui relient les doigts et les orteils et, en langage militaire, désigne une formation militaire en ordre serré ; la section est la coupe d'une forme géométrique pleine et marque le découpage hiérarchique du corps militaire; la colonne suit le même processus mais elle met l'accent sur la verticalité de la structure géométrique; la cohorte historiquement liée aux armées romaines désigne par extension aujourd'hui un groupe plus ou moins important; l'unité désigne l'articulation de plusieurs ensembles et, en ce sens, un bataillon, qui quantifie les soldats, est une unité tactique d'infanterie qui regroupe plusieurs compagnies; le même procédé s'applique à l'escadron, à la compagnie, au régiment, etc. La naturalisation est par conséquent le premier ancrage dans la culture institutionnelle, par le truchement du lien social et groupal en cours comme adhésion glissant vers 
l'expérience groupale où l'institution dépose, par l'intermédiaire des cultures militaires différenciées dans les corps, des attributs par attribution du corps militaire.

\section{Vers une incorporation de la guerre asexuée?}

Malgré un contexte de mutations organisationnelles et d'ouverture depuis la professionnalisation au vivier féminin, la littérature militaire et les textes réglementaires continuent à véhiculer une virilité guerrière, excluant ainsi $15 \%$ de ses effectifs. De plus, les femmes ne sont pas également représentées dans les corps militaires: plus de la moitié d'entre elles sont dans le service de santé des armées $(57,3 \%)$ et seulement $0,4 \%$ dans l'infanterie, à savoir une partie des troupes de mêlée qui interviennent en priorité sur un théâtre d'opérations (HCECM 2013: 26). Pour Françoise Héritier (2011:20-21), la question n'est pas celle de la force mais d'admettre l'idée que des femmes peuvent se battre, tuer, faire couler le sang et donc de faire couler un sang qui ne soit pas le leur. Les femmes sont toujours incluses dans une défense de la patrie par les armes où on défend un foyer, une famille dont elles sont les gardiennes. Dans les représentations masculines, elles ne peuvent donc pas être au cœur du combat car elles sont ce pour quoi ils se battent, un horizon étranger des champs de bataille. Dans une société agnatique qui recompose les liens de filiation, la hiérarchie militaire représente donc la figure paternelle et la patrie incarne la figure maternelle. Au temps du service national, les hommes incorporaient une identité militaire, une identité virile par la discipline du corps, en même temps que la nation et l'image d'une patrie à défendre par les armes par héritage révolutionnaire s'ancraient dans les collectifs (Boëne 2003 : 656). Le déliement de l'Etat-nation, l'affaiblissement de la patrie, la professionnalisation des armées ont laissé présager que les corps combattants pourraient dans le temps tendre vers une égalité de représentativité sexuelle où ce corps ne serait plus exclusivement masculin. Plus de vingt ans après la professionnalisation du corps militaire, les armées restent un monde d'hommes, et plus que jamais la Légion étrangère reste l'incarnation d'un corps militaire archétypal: professionnel avant la professionnalisation, attractif dans son recrutement et efficace dans ses missions, masculin sans intégration de femmes et donc sans «agent de déstructuration du collectif de travail masculin » (Fortino $1999: 376)$; sa seule pluralité est celle de la représentativité de la société qui garantit la légitimité des armées. À la Légion étrangère, le sismographe n'est pas national, il est international et suit les évolutions géopolitiques d'un monde en conflit permanent. Elle cultive à l'excès sa pluralité dont le seuil de tolérance reste consensuel: la pluralité rhizomorphique individuelle se fonde dans l'arbre-Légion où ce n'est pas seulement la dimension militaire qui compte mais l'aspect nomade (Deleuze et Guattari [1980] 1989: 453). Je lie ici les travaux de Deleuze et Guattari (1980) avec mon approche du légionnaire entre cohésion verticale (autorité hiérarchique) et horizontale (fraternité, camaraderie) à travers la métaphore filée de l'arbre où le légionnaire est présenté dans les discours des officiers comme un déraciné. Deleuze et Guattari distinguent ainsi l'arbre-racine qui est la démultiplication de l'unité, la racine-fasciculée qui nourrit la racine principale et le rhizome qui repose sur la pluralité et la capacité à se greffer non pas sur des racines mais sur d'autres rhizomes de manière horizontale. Le rhizome est alors alliance là où l'arbre est filiation. Les légionnaires construisent ainsi leur identité entre cette métaphore d'un ancrage à l'arbre-légion et l'incapacité à être l'arbre. En ce sens, ils sont rhizomorphiques et recomposent leur identité entre incorporation d'une identité 
professionnelle et parcours de vie incluant leur socialisation primaire qui ne peut être balayée: "Être rhizomorphe, c'est produire des tiges et filaments qui ont l'air de racines, ou mieux encore se connectent avec elles en pénétrant dans le tronc, quitte à les faire servir à de nouveaux usages étranges " (Deleuze et Guattari [1980] 1989: 23-24). En effet, les légionnaires, plus que les autres militaires, n'appartiennent véritablement à aucun territoire - de nombreux États interdisent l'engagement dans une armée étrangère et ces légionnaires prennent parfois le risque de devenir apatrides -, si ce n'est celui que reconstitue la Légion étrangère, comme nouvel ancrage identitaire. En s'effeuillant de leurs identités individuelles, ils abandonnent donc pour une identité collective un territoire, une filiation, une famille et parfois un nom, pour recomposer une nouvelle généalogie par greffe institutionnelle. Le corps sexué lui, est le degré irréductible d'un don total à une institution militaire qui n'a finalement aucun intérêt stratégique et opérationnel à recomposer une masculinité guerrière faite corps pour y intégrer une féminité guerrière. La pluralité des corps militaires continue ainsi à se fondre dans le creuset d'un unique corps combattant masculin dont les femmes militaires adoptent les codes et règles pour exercer leur métier des armes et ce, sans pouvoir toujours participer pleinement aux rites de cohésion qui forgent les liens entre militaires. La Légion étrangère n'est pas alors à considérer totalement comme un ultime bastion masculin. Elle est en quelque sorte le symbole cristallisé d'un monde professionnel en changement qui ne sait pas comment intégrer des femmes sans remettre en cause des siècles de construction d'un archétype guerrier qui n'a jamais été pensé autrement que comme masculin.

\section{BIBLIOGRAPHIE}

Audoin-Rouzeau, Stéphane et Becker, Annette, 2000, 14-18, retrouver la Guerre, Paris, Gallimard.

Benoît, Christian, 2014, « Du corps dans le langage des militaires », Corps, $\mathrm{n}^{\circ}$ 12, p. 127-129, [En ligne], https://www.cairn.info/revue-corps-2014-1-page-127.htm, consulté le 20 novembre 2019. DOI : 10.3917/corp1.012.0127

Boëne, Bernard, 2003, «La professionnalisation des armées : contexte et raisons, impact fonctionnel et sociopolitique ", Revue française de sociologie, vol. 44, p. 647-693, [En ligne], https://www.cairn.info/revue-francaise-de-sociologie-1-2003-4page-647.htm, consulté le 20 novembre 2019.

DOI : $10.3917 /$ rfs.444.0647

Bourdieu, Pierre, 1977, « Remarques provisoires sur la perception sociale du corps ", Actes de la recherche en sciences sociales, $\mathrm{n}^{\circ}$ 14, p. 51-54, [En ligne], https://www.persee.fr/doc/ arss_0335-5322_1977_num_14_1_2554, consulté le 20 novembre 2019.

DOI : $10.3406 /$ arss.1977.2554

Bouthoul, Gaston, 1970, Traité de polémologie, Paris, Payot.

COMLE, 2010, « Code d'honneur du légionnaire », [En ligne], http://www.legionetrangere.com/ $\mathrm{mdl} /$ info_seul.php?id=92\&idA=134\&block=6\&idA_SM=112, consulté le 20 novembre 2019. 
Deleuze, Gilles et Guattari, Félix, [1980] 1989, Capitalisme et schizophrénie, t. II, Mille plateaux, Paris, Minuit.

État-major de l'armée de Terre, 1985, Carnet de chants TTA 107, Conservatoire de musique militaire de l'Armée de Terre, approuvé sous le n 4159/DEF/EMAT/INS/FG/66 du 5 septembre 1985, annule et remplace la version de 1980.

État-major de l'armée de Terre, 2003, « Esprit de corps, traditions et identité dans l'armée de Terre », Paris.

Fortino, Sabine, 1999, « De la ségrégation sexuelle des postes à la mixité au travail : étude d'un processus », Sociologie du travail, vol. $41, \mathrm{n}^{\circ} 4$.

Gresle, François, 2003, « La "société militaire”. Son devenir à la lumière de la professionnalisation », Revue française de sociologie, vol. 44, p. 777-798, [En ligne], https:// www.cairn.info/revue-francaise-de-sociologie-1-2003-4-page-777.htm, consulté le 20 novembre 2019.

DOI : $10.3917 /$ rfs.444.0777

Guillaumin, Colette, 1992, Sexe, race et pratique du pouvoir. L'idée de Nature, Paris, Côté-femme.

Haut comité d'évaluation de la condition militaire (HCECM), 2013, Les femmes dans les forces armées françaises. De l'égalité juridique à l'égalité professionnelle, $7^{\mathrm{e}}$ rapport, Paris, La Documentation française.

Héritier, Françoise, 2011, « Entre culture et nature, Entretien avec Françoise Héritier », Inflexions, $\mathrm{n}^{\circ}$ 17, p. 19-30, [En ligne], https://www.cairn.info/revue-inflexions-2011-2-page-19.htm, consulté le 20 novembre 2019.

DOI : $10.3917 /$ infle.017.0019

Kaufmann, Laurence, 2006, « Les voies de la déférence. Sur la nature des concepts sociopolitiques ", Langage et société, $n^{\circ} 117$, p. 89-116, [En ligne], https://www.cairn.info/revuelangage-et-societe-2006-3-page-89.htm, consulté le 20 novembre 2019.

DOI : $10.3917 / 1$ s. 117.0089

Larroumet, Marie, 2004, Mythe et images de la Légion étrangère, Paris, L'Harmattan.

Lichtenberger, Yves, 2014, « Compromis et renoncement », Négociations, n² 22, p. 97-109, [En ligne], https://www.cairn.info/revue-negociations-2014-2-page-97.htm, consulté le 20 novembre 2019.

DOI : $10.3917 /$ neg.022.0097

Maisonneuve, Jean, 2018, La Dynamique des groupes, Paris, PUF, coll. « Que sais-je ? ».

Merleau-Ponty, Maurice, [1945] 1997, Phénoménologie de la perception, Paris, Gallimard.

Montagnon, Pierre, 2006, Légionnaires d'hier et d'aujourd'hui, Paris, Pygmalion.

Montety, Étienne de, 2006, Des hommes irréguliers, Paris, Perrin.

Moss, George L., 1997, L'Image de l'homme. L'invention de la virilité moderne, Paris, Éditions Abeille.

Paveau, Marie-Anne, 1999, «Images de la militarité dans les chants de l'Armée de terre française », dans A. Thiéblemont (dir.), Cultures et logiques militaires, Paris, PUF, p. 213-260.

Paveau, Marie-Anne, 2000, « Les frontières discursives de la militarité », Langage et Société, n 94 , p. 45-74, [En ligne], https://www.cairn.info/revue-langage-et-societe-2000-4-page-45.htm, consulté le 20 novembre 2019.

DOI : 10.3917/1s.094.0045 
Paveau, Marie-Anne, 2006, Les Prédiscours. Sens, mémoire, cognition, Paris, Presses Sorbonne nouvelle.

Périès, Gabriel, 1999, De l'action militaire à l'action politique : impulsion, codification et application de la doctrine de la " guerre révolutionnaire » au sein de l'armée française (1944-1960), thèse en science politique, Université Paris 1.

Pétonnet, Colette, 1982, «L'Observation flottante. L'exemple d'un cimetière parisien ", L'Homme, vol. 22, $n^{\circ}$ 4, p. 37-47, [En ligne], www.persee.fr/doc/hom_0439-4216_1982_num_22_4_368323, consulté le 20 novembre 2019.

DOI : $10.3406 /$ hom.1982.368323

Poussin, Adeline, 2014, Le chant militaire et sa pratique actuelle dans les Troupes de Marine, thèse d'ethnomusicologie, Université Nice Sophia-Antipolis.

Prévot, Emmanuelle, 2007, « Alcool et sociabilité militaire : de la cohésion au contrôle, de l'intégration à l'exclusion », Travailler, $\mathrm{n}^{\circ}$ 18, p. 159-181, [En ligne], https://www.cairn.info/ revue-travailler-2007-2-page-159.htm, consulté le 20 novembre 2019.

DOI : $10.3917 / \operatorname{trav} .018 .0159$

Reinert, Max, 1983, « Une méthode de classification descendante hiérarchique : application à l'analyse lexicale par contexte ", Cahiers de l'analyse des données, vol. 8, n 2, p. 187-198, [En ligne], http://www.numdam.org/item/?id=CAD_1983__8_2_187_0, consulté le 20 novembre 2019.

Thiéblemont, André (dir.), 1999, Cultures et logiques militaires, Paris, PUF.

Thuderoz, Christian, 2013, « Le problème du compromis », Négociations, n 20, p. 95-111, [En ligne], https://www.cairn.info/revue-negociations-2013-2-page-95.htm, consulté le 20 novembre 2019.

DOI : $10.3917 /$ neg.020.0095

Thura, Mathias, 2014, « “Dépêchez-vous d'attendre !" Travail militaire et socialisation au combat au sein d'une troupe de l'infanterie », Terrain, $\mathrm{n}^{\circ}$ 63, p. 54-72, [En ligne], http://

journals.openedition.org/terrain/15495, consulté le 20 novembre 2019.

DOI : $10.4000 /$ terrain.15495

\section{Corpus}

Service Information et Historique de la Légion étrangère, 1959, Marches et Chants de la Légion étrangère, Aubagne, Képi blanc.

Service Information et Historique de la Légion étrangère, 1974, Marches et Chants de la Légion étrangère, Aubagne, Képi blanc.

Service Information et Historique de la Légion étrangère, 1982, Marches et Chants de la Légion étrangère, Aubagne, Képi blanc.

Service Information et Historique de la Légion étrangère, 1985, Chants et Marches de la Légion étrangère, Aubagne, Képi blanc.

Service Information et Historique de la Légion étrangère, 1989, Chants et Marches de la Légion étrangère, Aubagne, Képi blanc.

Service Information et Historique de la Légion étrangère, 1993, Chants et Marches de la Légion étrangère, Aubagne, Képi Blanc. 
Service Information et Historique de la Légion étrangère, 1998, Chants de la Légion étrangère, Aubagne, Képi Blanc.

Service Information et Historique de la Légion étrangère, 2011, Chants de la Légion étrangère, Aubagne, Képi Blanc.

Entretiens menés par l'auteure de l'article auprès d'officiers de la Légion Étrangère, 2012 et 2016.

\section{NOTES}

1. Le roi Louis-Philippe crée la Légion étrangère par une ordonnance royale en 1831. Si elle fait partie de l'armée de Terre et est soumise au statut général des militaires de 2005, ses spécificités ont néanmoins fait l'objet d'un décret du Conseil d'État en 2008 (décret $\mathrm{n}^{\circ}$ 2008-956 du 12 septembre 2008 relatif aux militaires servant à titre étranger).

2. Certains postes sont encore fermés aux femmes pour des raisons spécifiques ou liés à des conditions d'exercice du métier comme dans les sous-marins nucléaires.

3. À ce sujet, voir Benoît (2014).

4. L'incorporation militaire correspond à la socialisation secondaire comme socialisation professionnelle. Cette incorporation débute à partir du moment où les soldats ont signé leur contrat et partent à l'instruction où ils apprendront le métier des armes, les distinctions entre les différentes cultures militaires, dont celle qui leur est plus particulièrement transmise - celle de leur arme -, et qui deviendra leur identité professionnelle. Les corps individuels y sont ainsi refondus pour s'ériger en corps professionnel marqué par la cohésion. Cette incorporation militaire se superpose de fait à une socialisation primaire. Mon point de départ a été cette incorporation militaire afin de définir ensuite le concept d'incorporation langagière qui englobe non seulement la dimension matérielle des corps mais aussi la dimension immatérielle recouverte par le langage et qui traverse les corps comme corps professionnel, corps individuel, corps collectif, corps langagier, etc.

5. Cette recherche est issue de huit ans de collaboration avec la Légion étrangère portée par un mémoire, puis un doctorat en cours.

6. J'indique la nationalité car ce sont les seuls chiffres dont nous disposons en l'absence de recensement linguistique.

7. Texte toutes armes.

8. IRaMuTeQ (Interface de $\mathrm{R}$ pour les Analyses Multidimensionnelles de Textes et de Questionnaires) est un logiciel libre qui reproduit la méthode de classification hiérarchique descendante $(\mathrm{CDH})$ de Reinert et qui est développé depuis 2008 par Pierre Ratinaud. Il permet des analyses textuelles par la statistique grâce aux langages $\mathrm{R}$ et Python. Constituer des segments de texte permet à IRaMuteQ de découper en unités plus petites un corpus pour l'analyser en fonction du nombre d'occurrences (ici 7 239), de lemmes (1 267) et de formes actives (1 064). Il constitue ensuite des classes selon le profil de ces segments qui révèlent le degré de dépendance d'une forme (ou lemmatisation) dans cette même classe. Le logiciel n'analyse pas en conséquent des mots isolés mais des segments (du cotexte) et permet ainsi de mettre en relief leur interdépendance (pourcentage, effectifs, chi2 d'association). Une forme peut donc apparaître dans plusieurs classes avec différents positionnements. Blanc par exemple 
dans un chant réfère à la neige mais la plupart du temps il est associé à képi pour former le segment képi blanc qui désigne par métonymie le légionnaire. Enfin, les pourcentages correspondent aux nombres de segments classés sur la totalité du corpus qui apparaissent dans la classe.

9. La classe 3 est celle de l'allégeance à la France ; la classe 4 renvoie à la transcendance et à la mort et la classe 2 à l'exaltation du sentiment d'appartenance.

10. Les signes de transcription utilisés visent à restituer les marques d'oralité du discours qui jalonnent la construction de celui-ci afin de contextualiser la teneur de l'interlocution. :/ indique ainsi un silence; ::: marque l'allongement prolongé d'une syllabe.

11. Ce chant apparaît dans les carnets de chants de la Légion étrangère depuis 2011 et l'extrait de ce couplet est chanté lors des popotes des officiers selon les indications du carnet. C'est pourquoi il est souvent absent des reproductions en libre accès des chants sur les sites consacrés à la Légion.

12. Par naturalisation, $j$ 'entends un processus éminemment social qui superpose un discours sur une nature humaine qui serait liée au sexage et donc à une fonction biologique d'un sexe à un discours de culture militaire qui retranche toute figure féminine d'une armée construite par des corps masculins et finalement pour des corps masculins. À titre d'exemple, on peut prendre en considération qu'aucune tenue militaire n'est adaptée pour une grossesse ou qu'un gilet pare-balle est conçu pour une musculature masculine.

\section{RÉSUMÉS}

Les représentations masculines en vigueur à la Légion étrangère déplacent la notion de patrie, non à celle de la France mais à la Légion étrangère, groupe d'appartenance dont la particularité est de recruter exclusivement des hommes. Par un processus d'incorporation, le corps masculin devient un corps collectif marqué par une masculinité guerrière dont la femme est exclue car étrangère. À partir d'un corpus de 57 chants tirés des carnets de chants édités par la Légion étrangère et d'entretiens réalisés avec des officiers de légion entre 2012 et 2016, je propose d'interroger ce corps collectif comme un corps discursif qui véhicule une naturalisation de rapports sociaux de travail en contexte militaire où s'exprime une virilité, une force qui entraîne une sexualisation des femmes comme objets langagiers. Dans un premier temps, je définis l'incorporation langagière comme un double processus de socialisation militaire. Dans un second temps, j'interroge le dépôt du corps individuel comme adhésion à un ordre militaire qui ouvre par la suite sur une exacerbation du collectif et une sexualisation du corps combattant. Enfin, je reviens sur l'incorporation d'une culture militaire qui revêt les formes d'une naturalisation du corps dans la guerre.

The masculine representations in force at the French Foreign Legion displace the notion of the fatherland, not that of France but the French Foreign Legion, a group of membership whose particularity is to recruit men exclusively. Through a process of incorporation, the male body becomes a collective body marked by a warlike masculinity of which the woman is excluded 
because foreign. Based on a corpus of 57 songs taken from the songbooks published by the French Foreign Legion and interviews conducted with legion officers between 2012 and 2016, I propose to question this collective body as a discursive body that conducts naturalization working social in a military context expressing virility, force that leads to the sexualization of women as language objects. At first, I define language incorporation as a dual process of military socialization. In a second time, I question the deposition of the individual body as an adhesion to a military order which opens later on an exacerbation of the collective and a sexualization of the fighting body. Finally, I return to the incorporation of a military culture that takes the forms of a naturalization of the body in the war.

INDEX

Mots-clés : masculinité, Légion étrangère, incorporation, guerre, corps discursif, naturalisation Keywords : masculinity, incorporation, French Foreign Legion, war, discursive body, naturalization

\section{AUTEUR}

\section{MÉLANIE TEXIER}

Université Rennes 2, PREFics (EA 7469) 